\title{
Objective analysis of contact lens fit
}

Authors: Lurdes Belda-Salmerón ${ }^{1}$; Tom Drew²; Lee Hall ${ }^{2,3}$, James Stuart Wolffsohn ${ }^{2}$

1. Optics Department, Faculty of Physics, University of Valencia, Spain

2. Ophthalmic Research Group, Aston University, Birmingham, UK

3. Visioncare Research, Farnham, UK

\section{Correspondence to:}

James S Wolffsohn.

Life and Health Sciences, Aston University, Aston Triangle, Birmingham, B4 7ET, UK.

Tel: $\quad$ +44 121204 4140; $\quad$ Fax: +44 1212044048.

e-mail: j.s.w.wolffsohn@aston.ac.uk

Running head: Objective versus subjective lens fit assessment.

Keywords: soft contact lens, fitting characteristics, lens evaluation, objective repeatability, validity.

The authors indicate no financial support or financial conflict of interest. All study procedures adhered to the recommendations of the declaration of Helsinki. Written consent was obtained from all participants. This research was supported in part by a Ministerio de Educación, Cultura y Deporte research scholarship to Lurdes Belda-Salmerón (FPU\#AP2010-2107\#).

\section{ABSTRACT}

Purpose: To assess the validity and repeatability of objective compared to subjective contact lens fit analysis.

Methods: Thirty-five subjects (aged $22.0 \pm 3.0$ years) wore two different soft contact lens designs. Four lens fit variables: centration, horizontal lag, post-blink movement in up-gaze 
and push-up recovery speed were assessed subjectively (four observers) and objectively from slit-lamp biomicroscopy captured images and video. The analysis was repeated a week later.

Results: The average of the four experienced observers was compared to objective measures, but centration, movement on blink, lag and push-up recovery speed all varied significantly between them $(p<0.001)$. Horizontal lens centration was on average close to central as assessed both objectively and subjectively $(p>0.05)$. The $95 \%$ confidence interval of subjective repeatability was better than objective assessment $( \pm 0.128 \mathrm{~mm}$ versus $\pm 0.167 \mathrm{~mm}, \mathrm{p}=0.417)$, but utilized only $78 \%$ of the objective range. Vertical centration assessed objectively showed a slight inferior decentration $(0.371 \pm 0.381 \mathrm{~mm})$ with good inter- and intrasession repeatability $(p>0.05)$. Movement-on-blink was lower estimated subjectively than measured objectively $(0.269 \pm 0.179 \mathrm{~mm}$ versus $0.352 \pm 0.355 \mathrm{~mm} ; p=0.035)$, but had better repeatability $( \pm 0.124 \mathrm{~mm}$ versus $\pm 0.314 \mathrm{~mm} 95 \%$ confidence interval) unless correcting for the smaller range (47\%). Horizontal lag was lower estimated subjectively $(0.562 \pm 0.259 \mathrm{~mm})$ than measured objectively $(0.708 \pm 0.374 \mathrm{~mm}, \mathrm{p}<0.001)$, had poorer repeatability $( \pm 0.132 \mathrm{~mm}$ versus $\pm 0.089 \mathrm{~mm} 95 \%$ confidence interval) and had a smaller range (63\%). Subjective categorization of push-up speed of recovery showed reasonable differentiation relative to objective measurement $(p<0.001)$.

Conclusions: The objective image analysis allows an accurate, reliable and repeatable assessment of soft contact lens fit characteristics, being a useful tool for research and optimization of lens fit in clinical practice. 


\section{INTRODUCTION}

There is a growing body of evidence to support the long-held clinical view that the assessment of lens fit is critical to contact lens practice. For soft contact lenses, this is limited to lens centration and corneal coverage, movement, surface wettability and subjective comfort. However, these characteristics in clinical practice are often recorded as acceptable ("good" fit) or unacceptable ("poor" fit) and may vary greatly between individual practitioners.(1) It is acknowledged that there are fewer lens parameters to consider when fitting soft lenses than with rigid lenses. Changes in lens fit cannot be predicted reliably by lens base-curve or material properties and vary between individuals. $(1,2)$ Nevertheless, attention to lens selection which includes lens material, dimensions and wearing modality, and accurate recording fit characteristics should not be ignored in order to ensure successful lens wear, avoiding future lens complications and to assist in overcoming contact lens discontinuations.(3)

Studies have shown that poor fitting soft contact lenses is commonly associated with discomfort,(4) poor vision(4) and drop out from wear(5) and it has a more negative impact on ocular physiology, as assessed by bulbar and limbal hyperaemia and corneal staining, compared to well-fitting lenses.(6) It is generally believed that lens mobility is correlated with the tear interchange, which is required to remove trapped debris, inflammatory cells and other tear components that would otherwise accumulate under the lens as well as to being necessary to provide sufficient oxygen levels at the tear-lens interface.(7) In the same way, the tear layer between the contact lens and cornea is also likely to reduce the friction between the surfaces, avoiding significant mechanical interaction, whereas the tear layer between the contact lens front surface and eye lid prevents tissue damage.(8)

Previous studies attempting to evaluate the relative importance of contact lens fit metrics have generally been subjective in nature, assessing features such as lens centration, movement on blink, lag and push-up, although the method employed was not always clearly articulated. $(4,9,10)$ A recent study assessing the impact of central and peripheral ocular surface shape on lens fit identified that the inherent variability of 
subjective lens fit was likely to have influenced the limited variability (24\%) that could be explained.(11) Moreover, it has also been demonstrated that the grading ability of even experienced eye-care practitioners is more variable and less sensitive than objective assessment,(12) although this has not yet been evaluated with lens fit metrics.

To overcome clinical bias and lack of precision, several studies have attempted to assess lens movement on blink objectively from video, but not all define the direction of gaze (primary or up-gaze), and other lens movements such as lag and push-up recovery speed have not been objectively evaluated.(13-16) Pritchard and Fonn(13) and Schwallie and Bauman(14) video recorded lens movement through a slit lamp and assessed centration and blink movement with a ruler used to make measurements off a monitor. A similar technique was used by Maldonado-Codina and Efron(15) after superimposing a projected gauge over the videos. Tranoudis and Efron(2) also used the same apparatus, but they adjusted the image to match an overlaid circle of known size to take measurements which additionally included up-gaze lag. However, the study by Wolffsohn and colleagues(1) was the first to make a comprehensive objective evaluation of lens fit in primary and multiple other positions of gaze, showing that movement on blink in up-gaze, horizontal lag and push-up recovery speed were the key metrics to independently characterize soft contact lens mobility.

Despite the increasing availability of digital capture through slit-lamp biomicroscopes, the validity and repeatability of objective lens fit analysis has never been determined. The aim of this study was to assess objectively soft contact lens fit characteristics and to determinate how objective analysis can provide the same key parameters as subjective evaluation, but with the advantages of being more repeatable, as well as having a higher resolution. 


\section{METHOD}

Thirty-five habitual contact wearing subjects (average $22.0 \pm 3.0$ years: $61 \%$ female) took part in the present study. The study was approved by the Human Sciences Ethical Committee and followed the tenets of the Declaration of Helsinki. Written informed consent was obtained from all subjects after receiving a full explanation of the nature and possible consequences of the study. All contact lenses used were commercially available and CE marked. Each subject was only included in the study if there was no evidence or history of binocular vision anomalies, or ocular disease including dry eye, or any pathology that would normally contraindicate contact lens wear. None of the subjects were on ocular medication.

The subjects, with a range of different corneal curvatures (horizontal meridian: $7.85 \pm 0.36 \mathrm{~mm}$; vertical meridian: $7.63 \pm 0.36 \mathrm{~mm}$; difference: $0.20 \pm 0.10 \mathrm{~mm}$ ), each wore two different soft contact lenses of power -2.50 diopters (D); a conventional hydrogel design (Vistakon Acuvue ${ }^{\circledR}$ 2, etafilcon A material, modulus $0.30 \mathrm{MPa}$ ) in one randomly assigned eye and a silicone-hydrogel design (Vistakon Acuvue ${ }^{\circledR}$ Advance $^{\circledR}$, galyfilcon A material, modulus $0.43 \mathrm{MPa}$ ) in the other (i.e. contralaterally). These lenses were chosen for their similar geometries and identical base curve $(8.3 \mathrm{~mm})$ and diameter $(14.0 \mathrm{~mm})$ parameters. The steepest available base curve $(8.30 \mathrm{~mm})$ was selected for dispensing in each case. The assessment of two contact lenses with different modulus allowed for a range of contact lens fit parameters that are commonly seen in clinical practice to be observed.

The study was designed as a randomized, double-blind, repeated measures study. Lens blister packs were re-labelled by a clinical assistant in order to ensure both investigator and subject were masked to lens type. After insertion by the masked investigator, at least thirty minutes to settle the contact lenses were given before assessment, a representative time of that of a lens settled after several hours of wear.(13, 16) The subject was asked to look straight ahead, then blink twice in primary gaze, look up and blink a further two times, look down while the upper lid was raised by the examiner to expose the superior lens edge and to look to the left and right. The lens was then 
pushed upwards digitally while the patient viewed in primary gaze so that the lower lens edge was raised to the middle of the cornea, before being released. The same experienced contact practitioner performed the whole routine on all the subjects. The assessment of lens fit was dynamically captured using a digital slit-lamp biomicroscope providing 6x magnification (CSO digital camera; resolution $1392 \times 1024$ pixels, frame rate $11 \mathrm{~Hz}$ ). The same resulting video footage was assessed for lens fit both subjectively and objectively to ensure a like-for-like evaluation of lens fit parameters.

\section{Subjective Lens Fit Analysis}

Four experienced investigators assessed four main lens fit variables: horizontal centration $(\mathrm{mm})$, post-blink movement in up-gaze $(\mathrm{mm})$, horizontal version lag (average of displacement of the lens from the primary position with nasal- and temporal-gaze; $\mathrm{mm}$ ) and push-up speed of recovery (slow/medium/fast) following digital displacement.(1) All observers repeated the analysis of all the subjects a week later.

\section{Objective Lens Fit Analysis}

A masked observer objectively analysed the resulting video using a purposedeveloped image analysis program (LabVIEW, National Instruments, Austin, Texas). Lens centration, both horizontal and vertical, was determined from the difference in millimetres between the centre of circles adjusted to circumscribe the visible limbus and contact lens edge in the horizontal and vertical axis, respectively. Movement on blink in up-gaze was assessed by the change in vertical lens position relative to the cornea from the first video frame after the blink until the lens was stabilized. Horizontal version lag was assessed as the difference in millimetres between the limbus to lens edge distance, from the primary gaze position to nasal- and temporal-gaze. Push-up recovery speed in millimetres per second was calculated from the change in vertical lens position relative to the cornea from immediately after the blink until it stabilized, divided by time (derived from the number of frames) taken for this to occur. The analysis was performed three times and repeated by the same masked researcher a week later. Imaging a graticule through the same slit-lamp and camera system determined the calibration as 1 pixel being equivalent to $0.016 \mathrm{~mm}$. 


\section{Statistical Analysis}

As the present study evaluated lens fit characteristics between techniques of assessment, rather than between eyes (different lens type in each eye), both eyes data was involved within the analysis. Objective data was considered in each session as the average of the three repeated measurements taken for each variable. Subjective data for lens fit characteristics was considered as the average across the four observers in each case. The difference in values between the objective and subjective techniques (validity) and, within (intra-session repeatability - difference between first and second measurement) and between sessions (inter-session repeatability) of assessing lens centration, movement on blink in up-gaze and horizontal lag was plotted versus the average and the Bland-Altman plotted for comparison. The Kolmogorov-Smirnov test was used to evaluate the normality of the data distribution with normally distributed data evaluated with parametric statistics. Hence objective versus subjective, intrasession and intersession differences were assessed by means of independent t-test and differences between observers assessed with related-samples Friedman's two-way Analysis of Variance by Ranks. The results were considered statistically significant when the $p$ value was less than 0.05. As push-up recovery speed could only feasibly be assessed as slow, medium or fast subjectively, a Spearman's rank non-parametric correlation with the objectively assessed recovery speed was conducted. All statistical analysis was performed using SPSS for Windows statistical software (version 20, SPSS, Inc.). 


\section{RESULTS}

\section{Validity}

\section{Lens centration}

Objectively measured centration was $0.371 \pm 0.381 \mathrm{~mm}$ vertically (inferior) and $0.067 \pm 0.211 \mathrm{~mm}$ horizontally (temporal) compared to the centre of the cornea (average $\pm \mathrm{SD}$ ). Subjectively estimated centration was $0.063 \pm 0.195 \mathrm{~mm}$ horizontally (temporal), varying between observers $(F=7.092, p<0.001)$, but could not be determined in the vertical direction due to the coverage of the lens periphery by the upper and lower eyelids. The mean difference between objective and subjective centration was $0.011 \pm$ $0.200 \mathrm{~mm}$ horizontally (average $\pm 95 \%$ confidence interval; Figure $1 \mathrm{~A}$ ). There was no significant difference between objectively and subjectively assessed horizontal centration $(p=0.417)$.

\section{Centration (Horizontal)}

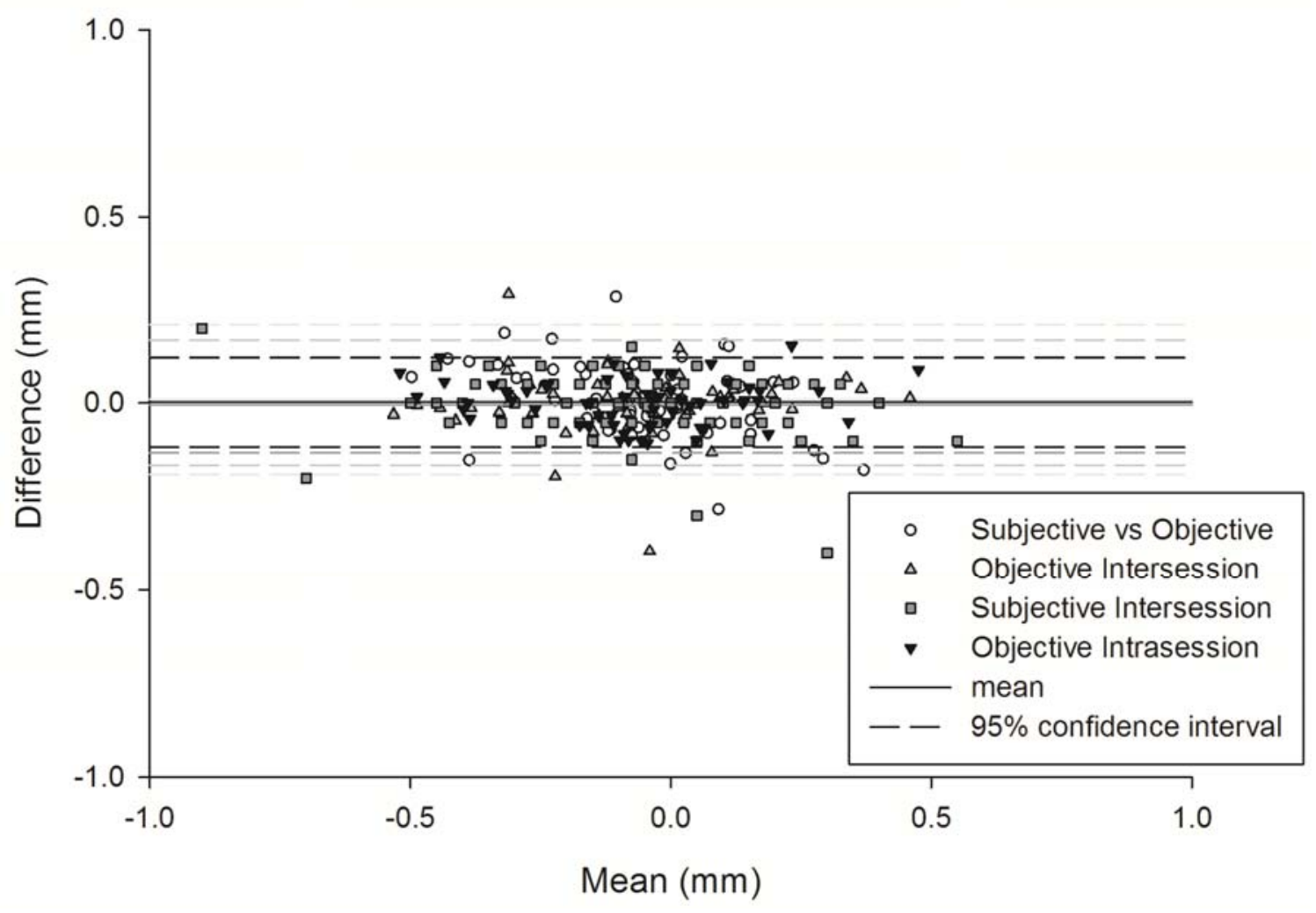

Figure 1. Bland-Altman plot of the difference in lens centration compared to the mean for subjective versus objective assessment (validity), objective repeatability both inter- and 
intrasession and subjective repeatability of horizontal centration (A) and objective repeatability both inter- and intrasession of vertical centration (B). Negative and positive values correspond to temporal and nasal decentration in horizontal centration $(A)$, and to superior and inferior decentration in vertical centration assessment (B), respectively. Solid line $=$ mean, dashed lines $=95 \%$ confidence interval. $N=70$ eyes .

\section{Movement on blink}

Movement on blink in up-gaze was $0.352 \pm 0.355 \mathrm{~mm}$ measured objectively and $0.269 \pm 0.179 \mathrm{~mm}$ estimated subjectively $(p=0.035)$, although there were significant differences between observers $(F=26.949, p, 0.001)$. The mean difference between objective and subjective movement on blink was $-0.070 \pm 0.512 \mathrm{~mm}$ (average $\pm 95 \%$ confidence interval; Figure 2).

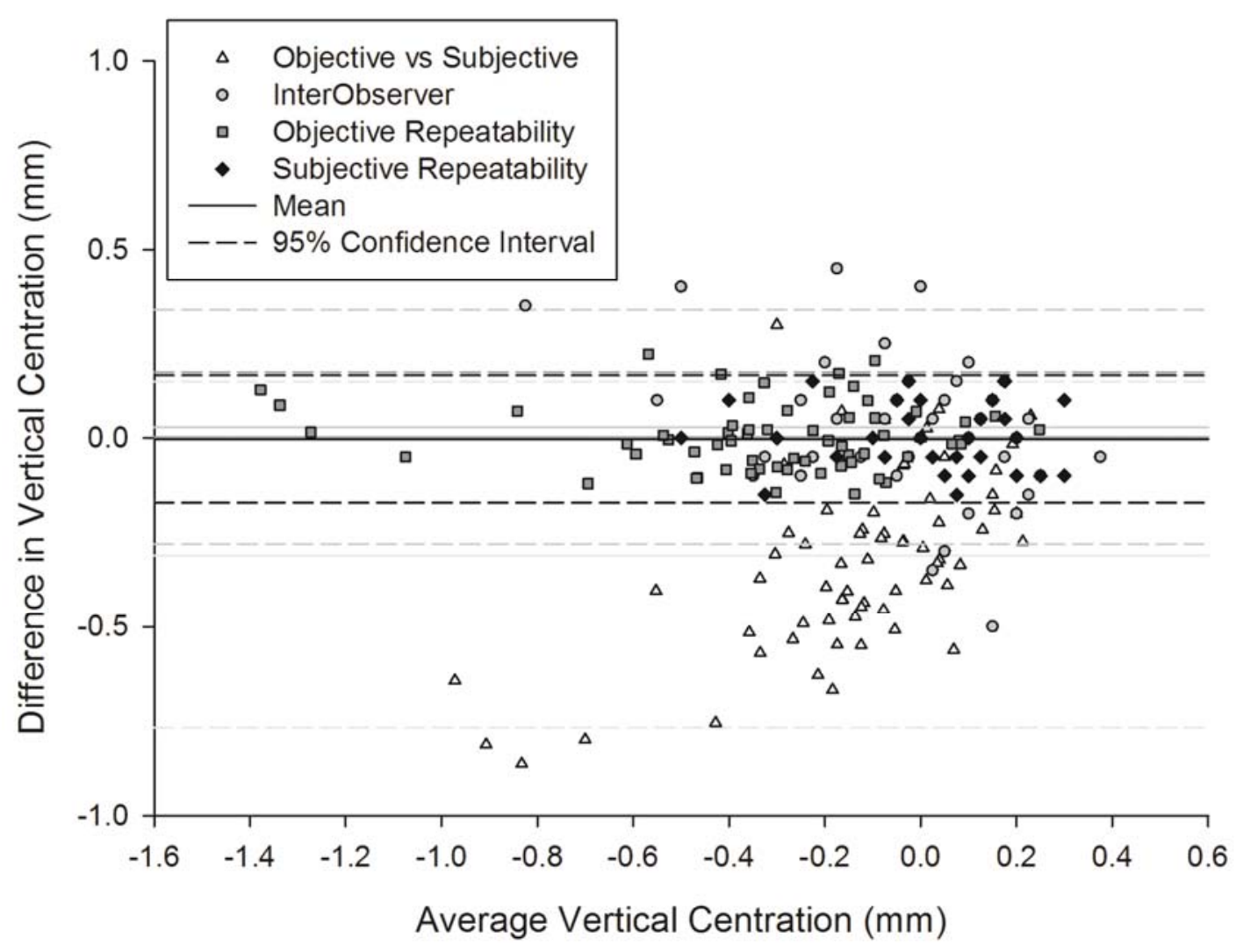

Figure 2. Bland-Altman plot of the difference in movement on blink compared to the mean for subjective versus objective assessment (validity), objective repeatability and 
subjective repeatability. Solid line $=$ mean, dashed lines $=95 \%$ confidence interval. $N=70$ eyes.

Lag

Horizontal lag considered as the average of nasal- and temporal-gaze lag values was greater measured objectively $(0.708 \pm 0.374 \mathrm{~mm})$ than estimated subjectively $(0.562$ $\pm 0.259 \mathrm{~mm} ; \mathrm{p}<0.001)$, and varied significantly between observers $(F=30.566, p<0.001)$. The mean difference between objective and subjective lag was $-0.148 \pm 0.342 \mathrm{~mm}$ (average $\pm 95 \%$ confidence interval; Figure 3 ).

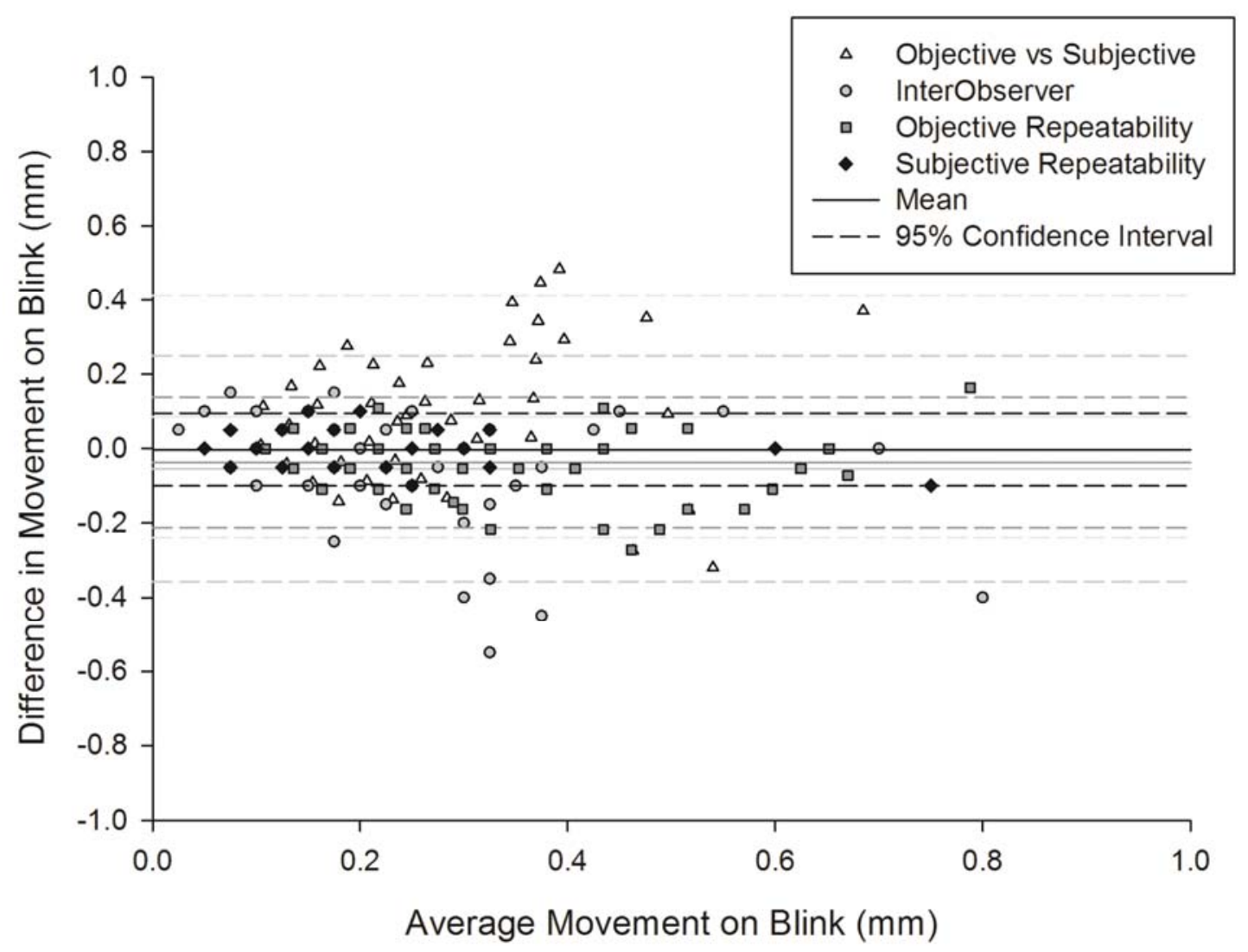

Figure 3. Bland-Altman plot of the difference in horizontal lag compared to the mean for subjective versus objective assessment (validity), objective repeatability both inter- and intrasession and subjective repeatability. Solid line $=$ mean, dashed lines $=95 \%$ confidence interval. $\mathrm{N}=70$ eyes. 


\section{Push-up Test}

Push-up speed of recovery was $0.491 \pm 0.213 \mathrm{~mm} / \mathrm{s}$ measured objectively. Subjective analysis by the four observers classified $22.5 \pm 23.2 \%$ as high mobility (1), $53.6 \pm$ $17.1 \%$ as medium mobility (2) and $23.9 \pm 7.6 \%$ as low mobility (3) for the original analysis and $20.0 \pm 17.8 \%$ as high mobility (1), $52.5 \pm 12.4 \%$ as medium mobility (2) and $27.5 \pm$ $8.9 \%$ as low mobility (3) a week later. There was a significant difference between the observers $(F=6.346, p, 0.001)$. The Spearman's rank correlation between objective and subjective push-up speed was $r=-0.493, p<0.001$ for observer 1 (red); $r=-0.472, p<$ 0.001 for observer 2 (green); $r=-0.361, p=0.005$ for observer 3 (purple) and $r=-0.351, p$ $=0.006$ for observer 4 (pink; Figure 4), with overall significance in objective scores between subjective grades $(F=31.850, p<0.001)$.



Figure 4. Box-plot of objective push-up recovery mean (line), standard deviation (box), 95\% confidence interval (bars) and outliers (dots) for subjective push-up recovery grades after the assessment by observers 1 to 4 (different shades of grey). $\mathrm{N}=70$ eyes. 


\section{Repeatability}

\section{Lens centration}

Objectively measured intrasession differences in centration were -0.004 \pm 0.175 mm vertically $(p=0.736)$ and $0.003 \pm 0.119 \mathrm{~mm}$ horizontally $(p=0.743)$ (average $\pm 95 \%$ confidence interval; Figure 1). Intersession repeatability was $0.012 \pm 0.139 \mathrm{~mm}$ vertically $(p=0.190)$ and $0.002 \pm 0.168 \mathrm{~mm}$ horizontally $(p=0.828)$ (average $\pm 95 \%$ confidence interval; Figure 1). Subjectively evaluated differences in centration for the four observers repeated measurement a week after the first assessment (intersession) were $-0.005 \pm$ $0.128 \mathrm{~mm}$ horizontally $(F=0.736, p=0.394$ ) (average $\pm 95 \%$ confidence interval; Figure 1A). The range of horizontal centration deviations was $0.532 \mathrm{~mm}$ (temporal) to $0.459 \mathrm{~mm}$ (nasal) with objective analysis. Approximately, $78.3 \%$ of this range $(0.463 \mathrm{~mm}$ to 0.313 $\mathrm{mm}$ for temporal and nasal deviation, respectively) was utilized in the subjective analysis.

\section{Movement on blink}

Objectively measured movement on blink intrasession differences in up-gaze between the first and second measurement was $0.018 \pm 0.152 \mathrm{~mm}(p=0.078)$ (average \pm 95\% confidence interval; Figure 2). The mean differences in movement on blink between the first and second analysis (intersession) were $-0.018 \pm 0.314 \mathrm{~mm}$ measured objectively $(p=0.379)$ and $-0.001 \pm 0.124 \mathrm{~mm}$ assessed subjectively across the four observers $(F=$ $0.001 ; p=0.999$ ) (average $\pm 95 \%$ confidence interval; Figure 2 ). The range of movement on blink was 0.020 to $1.966 \mathrm{~mm}$ with objective analysis and 0.100 to $1.013 \mathrm{~mm}$ rated subjectively ( $47 \%$ of the objective range).

\section{Lag}

Objectively measured intrasession differences in horizontal lag between the first and second measure was $-0.004 \pm 0.098 \mathrm{~mm}(p=0.500)$ (average $\pm 95 \%$ confidence 
interval; Figure 3). Objectively measured intersession differences in horizontal lag was statistically significant between the first and second analysis $(-0.017 \pm 0.089 \mathrm{~mm} ; \mathrm{p}=$ 0.007 ) (average $\pm 95 \%$ confidence interval; Figure 3 ). Subjectively differences in horizontal lag for the four observers repeated measurement a week after the first assessment (intersession) were $0.004 \pm 0.132 \mathrm{~mm}(\mathrm{~F}=1.275, \mathrm{p}=0.264)$ (average $\pm 95 \%$ confidence interval; Figure 3). The range of horizontal lag was 0.165 to $1.344 \mathrm{~mm}$ with objective analysis, but only $63.1 \%$ of this range $(0.269$ to $1.013 \mathrm{~mm}$ ) was utilized subjectively.

\section{Push-up Test}

Objectively measured difference in push-up recovery speed between the first and second analysis was $-0.005 \pm 0.084 \mathrm{~mm} / \mathrm{s}$ (average $\pm 95 \%$ confidence interval). No significant difference between the two objective analysis was found $(p=0.631)$. The range of objective push-up recovery speed was 0.184 to $1.245 \mathrm{~mm} / \mathrm{s}$. After subjective evaluation, only between $1.5 \%$ ( 1 eye) and 13\% (9 eyes) of observer's second assessments differed from their original grade. Therefore, Figure 4 presents push-up recovery speed outcomes only in the first analysis for each one of the four observers. 


\section{DISCUSSION}

The aim of the present study was to determine the validity and repeatability of objective lens fit analysis using images and video captured through a digital slit lamp as this has never previously been determined. Fitting soft contact lenses is a daily part of the workload of most optometrist. Thus, accurate recording fit characteristics needs to be performed in order to prevent future complications, such as higher levels of bulbar and limbal hyperaemia,(6) corneal staining,(6) tear exchange decrease,(7) or lens discontinuations(5) commonly related to poor lens fitting. It has been previously demonstrated that the grading ability of even experienced practitioners is more variable and less sensitive than objective assessment.(12) The hypothesis of present study was therefore that objective assessment of contact lens fit can provide the same key parameters as subjective evaluation, but has the advantages of being more repeatable as well as having a higher resolution.

Four main lens fit variables were assessed both objectively and subjectively. Lens centration assessment showed similar average results made horizontally for both objective and subjective measurements, and close to perfect centration relative to the limbus on average. The average of the four experienced observers was compared to objective measures, but centration, movement on blink, lag and push-up recovery speed all varied significantly between them, indicating the limitation of subjective grading both clinically and within research studies. The lenses centred slightly (on average $0.37 \mathrm{~mm}$ ) inferiorly relative to the limbus as might be expected due to gravity, although this differed from the previous objective lens fit paper where average vertical centration was almost central on average.(1) Precise vertical centration is difficult to evaluate subjectively without manipulation of the lids which could alter centration, with generally only the lower soft lens edge visible

Movement on blink in up-gaze was on average one third of a millimeter objectively, significantly higher than subjective estimation, but in both cases more than found in the previous study. It has been also demonstrated that horizontal lag was more predictive of overall lens movement than vertical lag or a combination of the two and 
hence this was assessed in the present study.(1) Horizontal lag considered as the average of nasal- and temporal-gaze lag values was greater measured objectively by about $25 \%$ compared to that estimated subjectively and again more than in the previous study. However, the settling time was longer in this study ( $>30$ minutes compared to 5 minutes and the lenses differed in design and materials. It is not feasible subjectively to simultaneously assess movement and time precisely; hence push-up recoveries were rated as slow, medium or fast, with over half assessed as falling in the middle category. Subjective categorization of push-up speed of recovery showed reasonable differentiation relative to objective measurement, although the variation of speeds within each classification was wide.

Repeatability was good both within and between sessions for objective measures. Interestingly, subjective measures appeared as repeatable as objective measures (albeit with lower precision as the minimum step sizes observers were able to grade to were larger). However, this was because the subjective range of measures was 47 to $78 \%$ of that of objective measurements.

In conclusion, the outcomes of the study suggest that objective image analysis allows an accurate assessment of soft contact lens fitting, being substantially more sensitive and more reliable than subjective grading. It should aid researchers to better understand the effect of lens design and material as well as corneal and peripheral topography on lens fit and may be developed further as a tool for use in clinical practice for optimization of lens fit, enabled by digital slit-lamp biomicroscopes or smartphone cameras.

\section{Acknowledgment}

The authors indicate no financial support or financial conflict of interest. All study procedures adhered to the recommendations of the declaration of Helsinki. Written consent was obtained from all participants. This research was supported in part by a Ministerio de Educación, Cultura y Deporte research scholarship to Lurdes Belda-Salmerón (FPU\#AP2010-2107\#). 


\section{REFERENCES}

1. Wolffsohn JS, Hunt OA, Basra AK. Simplified recording of soft contact lens fit. Contact Lens Ant Eye 2009;32:37-42.

2. Tranoudis I, Efron N. In-eye performance of soft contact lenses made from different materials. Contact Lens Ant Eye 2004;27:133-48.

3. Young G, Veys J, Pritchard N, Coleman S. A multi-centre study of lapsed contact lens wearers. Ophthalmic Physiol Opt 2002;22:516-27.

4. Young G. Evaluation of soft contact lens fitting characteristics. Optom Vis Sci 1996;73:24754.

5. Young G. Why one million contact lens wearers dropped out. Contact Lens Ant Eye 2004;27:83-5.

6. Young G, Coleman S. Poorly fitting soft lenses affect ocular integrity. CLAO J 2001;27:6874.

7. McNamara NA, Polse KA, Brand RJ, Graham AD, Chan JS, McKenney CD. Tear mixing under a soft contact lens: effects of lens diameter. Am J Ophthalmol 1999;127:659-65.

8. Korb DR, Herman JP, Blackie CA, Scaffidi RC, Greiner JV, Exford JM, Finnemore VM. Prevalence of lid wiper epitheliopathy in subjects with dry eye signs and symptoms. Cornea 2010;29:377-83.

9. Young G, Holden B, Cooke G. Influence of soft contact lens design on clinical performance. Optom Vis Sci 1993;70:394-403.

10. Morgan PB, Efron N. Comparative clinical performance of two silicone hydrogel contact lenses for continuous wear. Clin Exp Optom 2002;85:183-92.

11. Hall LA, Young G, Wolffsohn JS, Riley C. The influence of corneoscleral topography on soft contact lens fit. Invest Ophthalmol Vis Sci 2011;52:6801-6.

12. Peterson RC, Wolffsohn JS. Sensitivity and reliability of objective image analysis compared to subjective grading of bulbar hyperaemia. Br J Ophthalmol 2007;91:1464-6.

13. Pritchard N, Fonn D. Dehydration, lens movement and dryness ratings of hydrogel contact lenses. Ophthalmic Physiol Opt 1995;15:281-6.

14. Schwallie JD, Bauman RE. Fitting characteristics of Dailies daily disposable hydrogel contact lenses. CLAO J 1998;24:102-6.

15. Maldonado-Codina C, Efron N. Impact of manufacturing technology and material composition on the clinical performance of hydrogel lenses. Optom Vis Sci 2004;81:442-54. 
16. Brennan NA, Lindsay RG, McCraw K, Young L, Bruce AS, Golding TR. Soft lens movement: temporal characteristics. Optom Vis Sci 1994;71:359-63. 